\title{
Verrucous nipples
}

A 29-year-old woman (gravida 3, parity 3) at 20 weeks of gestation presented with skin growth at bilateral nipples and areola for past 5 years. It was first noticed during her first pregnancy. The lesions were gradually increasing in size, otherwise asymptomatic. Previously, she had difficulty to breastfeed twins from her second pregnancy. She has no known medical illness. She denied personal and family history of atopy. Physical examination revealed verrucous hyperpigmented plaques on bilateral areola and nipples. No genital or mouth lesion was seen.

What is the most likely diagnosis?
A. Eczema of the nipple
B. Hyperkeratosis of nipple and areola
C Mammary Paget's disease.
D. Papillomatosis of the nipple
E. Viral warts

Diagnosis. Nipple eczema is a dermatitis involving the nipple and areola, and is characterised by oozing, crusting and scaling. Absence of personal and family history of atopy makes the diagnosis of eczema unlikely. Additionally, the lesion is relatively asymptomatic as pruritus is a key symptom in eczema.

Mammary Paget's disease is a rare cutaneous malignancy that is associated with breast carcinoma. It usually occurs in patients between the ages of 50 and 60 years. Mammary Paget's disease is characterised by chronic eczematous lesion where it may look erythematous, with scaling, swelling, ulcerating and oozing. The diagnosis is confirmed by skin biopsy.

Nipple papillomatosis is a benign growth of numerous papillomas from the lactiferous duct epithelium of the nipple. It is commonly diagnosed in women aged 35 to
55 years old. It is usually asymptomatic, though patients can experience mild tenderness from it. The diagnosis is confirmed by a skin biopsy.

Viral warts are caused by infection with human papillomavirus resulting in benign proliferations of the skin and mucosa. It commonly presents as hyperkeratotic and verrucous papules. Common affected sites are hands, feet, face and genitalia. Dermoscopy and skin biopsy can help to confirm the diagnosis.

This woman had secondary hyperkeratosis of the nipple and areola. It can occur in puberty and pregnant women. She started having the lesion during her first pregnancy. The histopathological findings confirmed the diagnosis (Figs. 2A to 2C). From the skin biopsy, epidermal acanthosis with thickened epithelium predominantly composed of basaloid cells was seen in the epidermis. There were interspersed horn cysts. The dermis showed minimal perivascular lymphoplasmacytic infiltrates, no atypia or evidence of malignancy.

Discussion. Hyperkeratosis of the nipple and areola is a rare condition with unclear pathogenesis. Despite its rarity, its classification has been revised twice. The latest classification suggested by Pérez-Izquierdo divides hyperkeratosis of the nipple and areola into 2 types: (1) the primary or idiopathic type that typically presents in adolescent females, and (2) the secondary type associated with another skin condition, which can be unilateral or bilateral depending on the underlying dermatoses. ${ }^{1}$

Unilateral secondary hyperkeratosis of the nipple and areola may occur in seborrhoeic keratosis, papillomatous melanocytic naevus, epidermal naevus, leiomyoma, acanthosis nigricans, nipple papillomatosis,

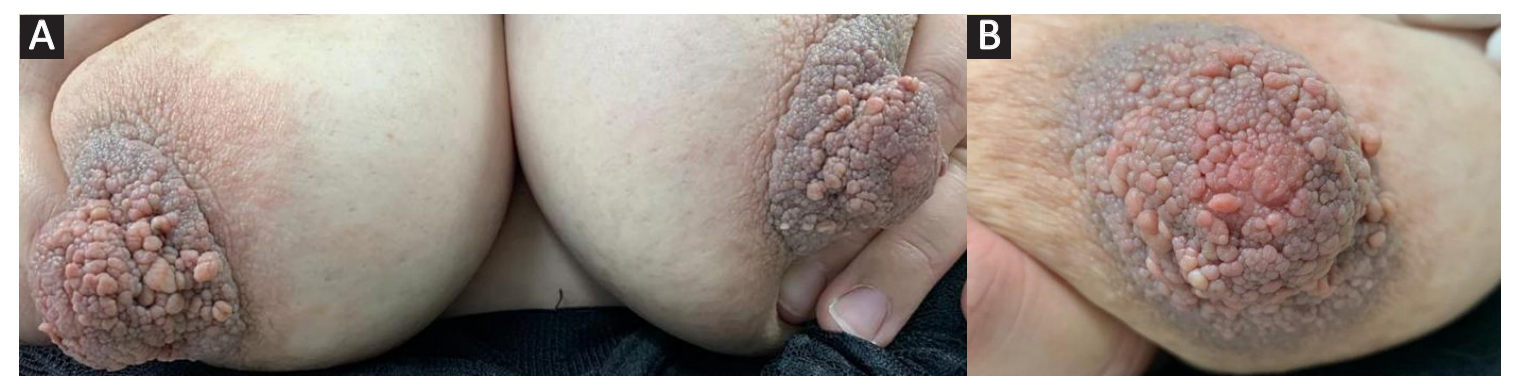

Fig. 1. (A and B): Verrucous hyperpigmented plaques on bilateral areolae and nipples. 

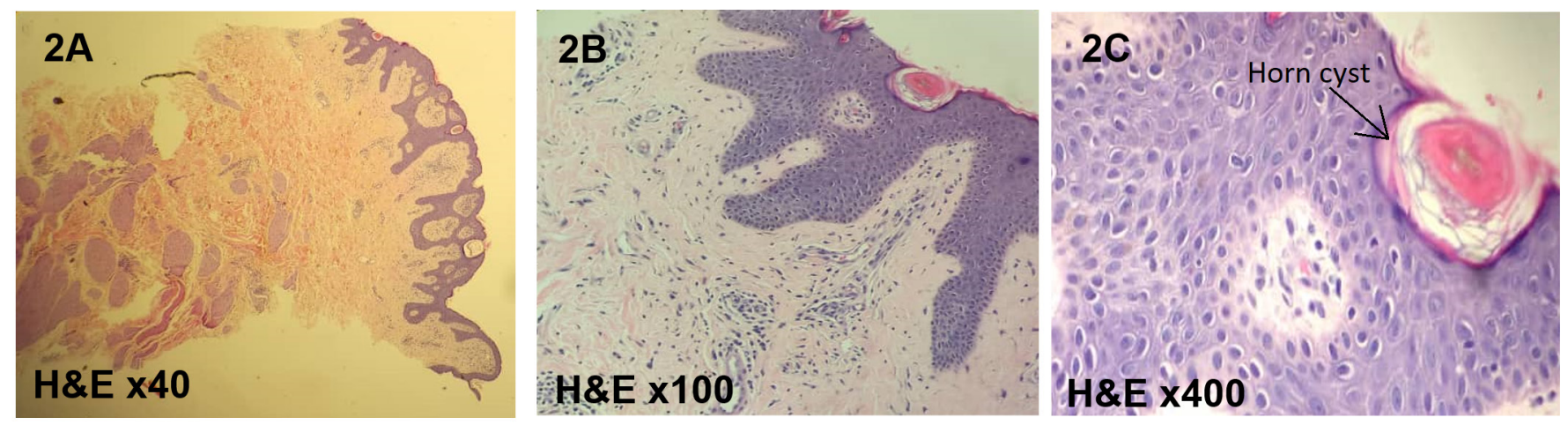

Fig. 2. (A): Thickened epithelium with acanthosis at low power magnification. (B) and (C): Epidermis has predominantly basaloid cells with horn cyst (see arrow) at higher power magnification.

jogger's or cyclist's nipple, mammary Paget's disease of the skin, and skin cancer. Bilateral secondary hyperkeratosis of the nipple and areola may occur with ichthyosis, atopic dermatitis, psoriasis, Darier disease and targeted cancer therapies such as vemurafenib. Breast lesions developed during pregnancy and in males receiving oestrogen therapy are also included as secondary hyperkeratosis of the nipple and areola. ${ }^{2}$

There is no definitive treatment for hyperkeratosis. Clinically proven topical therapeutic options include keratolytic (salicylic acid, 6\%), emollients, corticosteroids, tretinoin, and calcipotriol. Interventional treatments such as shave excision, cryotherapy and carbon dioxide laser have also been reported as options. ${ }^{3}$

For the patient's third pregnancy, she considered breastfeeding by extraction of breastmilk (as she did for her second pregnancy). She was concerned about wound healing from surgical excision affecting the extraction of breast milk, and vice versa. Thus, she declined surgical treatment initially. However instead of breastfeeding, she started her child on formula milk after delivery. In the last follow-up, she expressed her readiness for surgical excision.

Conclusion. Hyperkeratosis of the nipple and areola is a rare and benign condition. It does not resolve without treatment and may recur after treatment. It is usually cosmetically unacceptable and may cause psychologic disturbances in affected persons. Functionally, it can interfere with breastfeeding.

\section{REFERENCES}

1. Shastry V, Betkerur J, Kushalappa PA. Unilateral nevoid hyperkeratosis of the nipple: A report of two cases. Indian J Dermatol Venereol Leprol 2006;72:303-5.

2. Pérez-Izquierdo JM, Vilata JJ, Sánchez JL, et al. Retinoic Acid Treatment of Nipple Hyperkeratosis. Arch Dermatol 1990;126:687-8.

3. Wang MF, Wang L, Li LF. Bilateral hyperkeratosis of the nipples and areolae with linear nevus: a rare case report and review of the literature. Postgrad Med 2018;130:361-4.

Jin Huang Lim, ${ }^{1} M R C P(U K)$, Kwee Eng Tey, ${ }^{1} M R C P(U K)$

${ }^{1}$ Department of Dermatology, Hospital Sultanah Aminah, Johor Bahru, Malaysia

Correspondence: Dr Jin Huang Lim, Department of Dermatology, Hospital Sultanah Aminah, Jalan Persiaran Abu Bakar Sultan, 80000 Johor Bahru, Malaysia.

Email: limjinhuang@gmail.com 